repcated. We have effectively repeated this procedure five and six times at short intervals. Up to now we have chiefly used the method in cases where the ordinary nonoperative treatment was practically ineffective and in which we desired to get the eye into a more favourable state for operation. It is not our object in this communication to do more than draw attention to the frequently favourable results of the adrenaline pack on the tension in glaucoma of moderate extent. At the same time, we would like to show some effects of consiclerable theoretical interest in connexion with its use in very high tension cases.

\section{The Method.}

The eve is anaesthetized by two or three instillations of 2 per cent. holocaine. The tension is taken with an instrument (we usually use a Bailliart's tonometer) if it is desired to make accurate chart records. If we wish to try and obviate the initial rise of tension due to the adrenaline it is desirable to give eserine frequently before and after the pack-say, six instillations in the hour before, and six half-hourly instillations after. A small flattened pledget of wool is soaked in ordinary adrenaline chloride solution ( 1 in 1,000, P., D. and Co.), and whilst the patient looks down and the lid is elevated with a retractor, it is laid in the superior fornix and the eyc closed. It is left there for five minutes. Should eserine not be used in conjunction with the adrenaline the tension is more likely to rise, but eren with marked rises we have not scen an untoward result; there has always been a fall again the following day. In the ordinary case of primary glaucoma the reactions are moderate, and may be slight and hard to demonstrate with the tonometer, although the fall is usually definite.
In badly damaged eyes with excessive tensions, however, more startling effects may be obtained, some of which are quite contrary to what might be expected, and more interesting in view of the fact that the vascular tunic in such eyes is markedly atrophic. A few selected charts will better illustrate various responses to the adrenaline pack.

Chart 1.--A case of moderate tension in which there was no rise immediately after the pack on the first occasion and actually a fall on the second. Eserine was freely instilled as described above, before and after the pack. Operation. was undertaken before the fall due to the second pack had passed. Escrine alone at first reduced the tension, but it rose on the sixth day.

Chart 2 shows the effect of numerous packs in a case where there was high tension in both eyes. The first pack in the right eye acted well on both, but the second pack, given in the left eye, did not reduce the tension in the right. Thereafter packs were given on both sides. The last packs were not very effective.

Chart 3 shows marked rises immediately after the packs in an eye of moderate tension. The value of the pack in such a case would appear to be doubtful. Small corresponding rises are shown in the untreated eye, which was apparently normal,

Chart \& shows a marked late fall of tension due to the adrenaline pack without an immediate rise. The tension in the other eye, which did not get adrenaline, shows corresponding variations of tension. The fall due to the pack (in spite of eserine) is not well maintained after the first pack. It is of somewhat longer duration after the second. The patient was eventualiy treated by operation.

Chart 5 shows the effect of the pack on an ere of very high tension. The riscs immediately following the pack are small; the falls are comparatively small and of short duration. $A$ curious feature is the effect on the opposite eye, where the tension behaves in a converse manner to that in the treated eye.

Chart 6 shows the effect of eserine and the adrenaline pack with corrasponding falls in the untreated eye. Eventually, after. a number of badly sustained falls, a low-level tension of longer duration was obtained.

\section{THE ACETIC ANHYDRIDE TEST OF THE SPINAL FLUID.}

BY

G. L. PIOTROWSKI, M.D.Geneva, M.R.C.S., L.R.C.P., PRIVAT-DOCENT AT TTE FACULTY OF MEDICINE OF GENEVA.

J. G. Greenfield and E. A. Carmichael have investigated the value of the acetic anhydride test. The reaction was found to be positive in 15 out of 16 cases of dementia paralytica, and a positive reaction might also-occur in secondary neurosyphilis; cases of cerebral degeneration were said to give a weak reaction. Greenfield and Carmichael suggested that the reaction might be due to an increased cholesterol'content of the spinal fluid.

I lave investigated the acetic anhydride test in 88 cases, and give here my first impression on its value. When the investigation could not be undertaken at once we always kept the fluid in the ice-chest, but never waited more than twenty-four hours. One cubic centimetre of fluid is measured in a test tube, and $0.3 \mathrm{c.cm}$. of acetic anhydride is added; it is then shaken so as to produce an emulsion, and then, drop by drop, $0.8 \mathrm{c.cm}$. of pure sulphuric acid is introduced. As we often proved, it is of the utmost importance to add the sulphuric acid very slowly; a negative fluid may give a positive result if the sulphuric acid is run in too quickly. In the case of a positive reaction a lilac tint of varying shades appears, but in my opinion is not so fleeting as the authors describe it.

The numeration of the cells of the cerebro-spinal fluid was made with the Nageotte's counting chamber, and the quantity of albumin estimated with the Sicard and Cantaloube process (presipitation by trichloracetic acid); the globulins were detected by the Nonne-Apelt and the Pandy tests. The following are the results in a few diseases.

(iencral Paralysis of the Insane. (10 cases).-No relation was found between a positive Wassermann reaction and a positive acetic anhydride reaction, and no association between it and the presence of globulins. In the four negative cases the number of cells per cubic millimetre was always below 3 , and the albumin below 0.035 per cent. The acetic ankydride test was strongly positive in two cases, and positive in four patients; in all these cases the cell count was more than 20 cells per cubic millimetre.

Tubes (5 cases). - None of the cases we investigated had more than 5 cells per cubic millimetre in the spinal fluid, and all gave negative test.
Mcningitis (15 cases).-In 6 cases of tuberculous meningitis we had 4 positive reactions and 2 negative; of these last cases one contained 37 cells per cubic millimetre and the other 15 cells, with an albumin content of 0.044 and 0.025 per cent. In three of the positive cases the diagnosis was confirmed by a post-mortem examination. In one case of staphylococcal meningitis of unknown origin (the patient was comatose when admitted to the hospital), with thrombosis of the carernous sinus, there was a strongly positive acetic anhydride reaction, the spinal fluid being almost pure pus. In two other cases of meningitis there wcre positive reactions; in one of these an abscess of the temporal lobe was found at the necropsy, but no organism could be found, and the origin remained unknown. In these three cases the albumin was as high as 2.0 per litre, and the cells were innumerable.

Syphilitic Mcningitis and Ccrcbral Luas.-A negative test was obtained in an old-standing case, treated when the cerebro-spinal fluid was normal; in two other cases the test was doubtful. Cytological examination of the spinal fluid revealed 6 and 16 cells per cubic millimetre; 0.025 to 0.035 per cent. of albumin was present, and the Wassermann reaction was positive. In the other cases the test was strongly positive; the meningeal reaction was also positive, there being 80 to 310 cells per cubic millimetre.

Congcnital Syphilis.-Two cases, with a negative fluid and a negative test.

Mcningcal Hacmorrhage (4 cases).-Negative test in all; the spinal fluid contained blood.

Cercbral Degcncration (10 cases).-We could only investigate the arhydride acetic test in 10 cases; the number of cclls was never above the usual figure, and the quantity of albumin was always normal. In seven patients in whom degeneration was marked there was a positive or a subpositive reaction.

Cercbral Tumours.-The test was always negative; none of our cases had more than 3 cells per cubic millimetre.

Epidemic Encephalitis (5 cases).-The reaction was always negative, although the number of .. cells was three times above the normal (up to 27).

Zona.-Three cases, always negative results, and slight meningeal reaction.

Poliomyclitis.-Two cases, each reacting slightly positively.

Various Cascs.-In 20 other cases there were 9 of meningism (fracture of the skull, headache, neurasthenia, hysteria). In 5 a weakly positive reaction was found, although there was no question of cercbral degeneration or of syphilis. In all these cases the spinal fluid and the results of the clinical examination were completely normal.

If we except the cases with a slight positive reaction we only get a positive test in general paralysis, in meningitis, and in cerebral degeneration. There is a certain relation between the number of cells per cubic millimetre and a positive reaction; the nature of the colls seems to have 
no definite significance, fluids with lymphocytes giving a positive reaction as well as fluids with polymorphonuclears.

We estimated the cholesterol content of the spinal fluid in 15 cases, but the quantities found were even smaller in cases with a positive acetic anhydride test. The addition of cholesterol does not transform a negative test into a positive one. We do not think it is possible to find the explanation of the acetic anhydride test in the presence of cholesterol.

Is the acetic anhydride test to be correlated with the presence of cholin? Kinnier Wilson found cholin present in cases with degeneration of the central nervous system. Guggenheim and Loeffler found also an increased quantity of cholin in the spinal fluid of syphilitic patients (cerebrospinal syphilis, tabes). We are not able to answer that question, since the quantity of spinal fluid at our disposal was never sufficient to allow us to determine the presence of that substance; but Kaufmann, working on large amounts of spinal fluid, has demonstrated that normal and pathological fluids contain traces of a base which is certainly not cholin (betain?).

We think the positive acetic anhydride test depends on the presence of a substance (cerebroside) secreted or due to the cytolysis of the leucocytes or of the nervous tissues. A drop of sterile pus added to a negative spinal fluì gives a positive test (pyosin). An emulsion in normal saline of liver, spleen, and brain gives a strong positive reaction, especially the liver, which is well known to contain a large quantity of lipoids. With glycocoll and lecithin the acetic anhydride test remained negative.

We are still in doubt as to the exact cause of the positive acetic anhydride test, which is due, in our opinion, to a cerebroside of the type of pyosin.

If we except the cases with a strong cytological reaction, we found a positive test in general paralysis and cerebral degeneration, and we suggest that the acetic anhydride test might be used as an argument in the differential diagnosis between cerebral degeneration and haemorrhage into the brain, or at least to detect a disintegration of the central nervous system. The acetic test is only of value in the absence of any meningeal reaction of the spinal fluid.

Greenfield, J. G., and Carmichael, E. A.: Journ. Neurol. and Psychopathol., January, 1927, p. 220.

Guggenheim and Loeffler : Biochem. Zeit., 74, 1916, 208.

Kinnier Wilson: Rev. Neurol, 1904, 401.

Kaufmann: Zeit. f. Phys. Chemie, 66, 1910, 343.

\section{Ettemuranda: \\ MEDICAL, SURGICAL, OBSTETRICAL.}

\section{ABDOMINAL TORSION OF THE OMENTUM.}

The following very interesting case of abdominal torsion of the omentum occurred in my general practice early this year.

The patient, a man aged 39, was admitted to the Dunfermline and West Fife Hospital on February 22nd with the following history.

For the past six months he had had abdominal symptoms, and three months before his admission $x$-ray examination suggested the diagnosis of duodenal ulcer. A month later he had an attack of pain in the right hypochondrium, accompanied by a 1 ise of temperature; the attack lasted four days. Since then he has had two similar attacks, which were treated by his doctor as acute appendicitis. On February 20th he was seized with severe epigastric appendicitis. On February 20 six hours after its onset the pain pain and vomited to the right hypochondrium, and remained there till shifted to the right hypochondrium, and remained there till he had been given an enema with gocd result, but without effect on the pain. On examination, the patient, a fat, flabby man, was seen to be suffering acutely; his tongue was clean and moist, his temperature $101^{\circ} \mathrm{F}$, and his pulse rate 108 . The abdomen was distended, inumobile, and rigid and tender all over: the maximum tenderness was in the right iliac fossa. The percussion note was tympanitic throughout, and the liver dullness was diminished. The signs suggested either ruptured appendix or ruptured duodenal ulcer.

Oniration.-The abdomen was opened by a two-inch midline incision above the pubis, and a suction tube was inserted into the abdominal cavity; no fluid was encountered, nor was there any in the pelvis. An incision was then made along the outer side of the right rectus. The caecum was very distended but the appendix was normal ; the transverse colon, too, was greatly distended, but no colonic obstruction could be discovered. The appendix was removed, and the incision then extended upwards.
The gall-bladder was normal, but a "doughy" mass, lying high up in the right: hypochondrium, was delivered without difficulty. It was of a dark plum colour, and consisted of the great omentum twisted on itself six times. The omentum was removed proximally to the twist, and though the abdomen was further palpated for obstruction of the colon nome could be found. The duodenum obstruction of the colon norre could be found. The duodenum was palpated, but not seen. On account of the intense distension and thinning of the caecum a No. 12 catheter was
inserted into it by Senn's method and brought out through a stab wound in the right iliac fossa.

The patient made an uninterrupted recovery, and was discharged on March 7th.

The removed omentum measured $7 \mathrm{in}$. by $3 \mathrm{in}$. Its pedicle consisted of six twists, of which four could be undone easily. The omental veins were greatly distended.

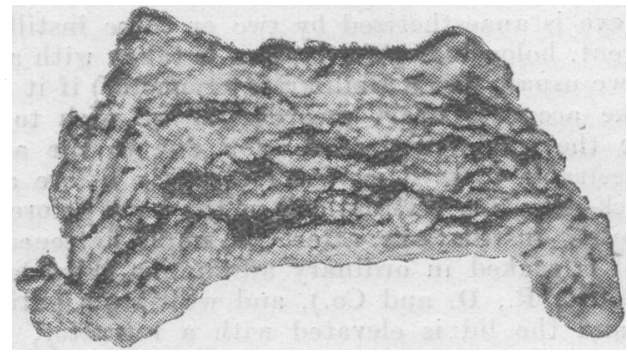

The following are interesting features in this case.

1. A true primary torsion of the omentum appears to have been present; there was no hernia, the appendix was normal, and there were no adhesions.

2. The previous attacks of right-sided abdominal pain with temperature might have been due to an inflammatory state of the omentum preceding the torsion, and a similar explanation might be advanced for the symptoms of duodenal ulcer.

3 . The absence of blood-stained fluid in the peritoneal cavity is noteworthy. In the British Journal of Surgery of April, 1925 (p. 738), Mr. Ernest. Cowell stated that " a rush of blood-stained fluid on opening the abdomen" should remind one of the possibility of omental torsion. The marked distension of the colon, a form of reflex ileus due to irritation of the splanchnic nerves involved in the torsion, has, so far as I know, not been described in other cases.

I am indebted to Mr. Peter Christie of Dunfermline for the photograph.

\section{J. M. Black, M.B., Ch.B., F.R.C.S.Ed., Honorary Surgeon, Dunfermline and West Fife Hospital.}

\section{GRAVITATIONAL MASSAGE.}

THe patient, a medical man aged 74 , has suffered from severe dyspeptic symptoms continuously for two years, and before that intermittently. Till May this year he had a very poor appetite, and taking food caused considerable pain. He had lost weight markedly, looked sickly, suffered from depression, and had been obliged to give up work. He attributed his condition to previous overfeeding. For some years he had lost the power of vomiting; even when he felt the subjective symptoms of sea-sickness vomiting did not occur. Early in May I became aware that a mass was retained in his sagging stomach. Abdominal massage produced no relief, and it seemed that failure to shift the mass was due to the fact that it was retained by gravitation. Treatment by gravitational massage was therefore tried, and attended by immediate success.

The method adopted was as follows. The patient lay in bed on his back, resting his fcet on an article of furniture 3 feet higher than the surface on which he was lying. By flexing his knees and working his head nearer the article of furniture he was able to place his trunk in an almost vertical position, with the result that his stomach changed its position in relation to the pylorus. On performing abdominal massage $I$ at once felt the lump slip down, apparently into the duodenum, and the patient was conscious of immediate relief. Since the regular adoption of this treatment all acute pain has ceased, and the dyspeptic symptoms-as, for example, aversion to food, bad taste in the mouth, mental depression and sleeplessnesshave subsided. The patient's healthy colour, bodily vigour, and normal weight have returned.

Shrewsbury.

A. G. Mackenzie, M.D, 